Acta Crystallographica Section E

Structure Reports

Online

ISSN 1600-5368

\section{(E)-5-[(1,5-Dimethyl-3-oxo-2-phenyl-2,3- dihydro-1H-pyrazol-4-yl)iminomethyl]-2- methoxyphenyl 4-chlorobenzene- sulfonate}

\section{Tian-Xiang Lei}

Department Of Electrical Engineering, North China Electric Power University, Baoding City, Hebei Province 071003, People's Republic of China

Correspondence e-mail: lei_tianxiang@163.com

Received 6 June 2012; accepted 10 June 2012

Key indicators: single-crystal X-ray study; $T=294 \mathrm{~K}$; mean $\sigma(\mathrm{C}-\mathrm{C})=0.004 \AA$; $R$ factor $=0.056 ; w R$ factor $=0.154 ;$ data-to-parameter ratio $=13.5$.

In the title compound, $\mathrm{C}_{25} \mathrm{H}_{22} \mathrm{ClN}_{3} \mathrm{O}_{5} \mathrm{~S}$, the two $\mathrm{N}$ atoms in the pyrazole ring have a pyramidal environment, with the sums of the valence angles around them being 349.3 (2) and $357.5(2)^{\circ}$. The phenyl ring is twisted by $50.97(12)^{\circ}$ from the pyrazole mean plane. In the crystal, pairs of weak $\mathrm{C}-\mathrm{H} \cdots \mathrm{O}$ hydrogen bonds link the molecules into inversion dimers.

\section{Related literature}

For general background to the use of Schiff base derivatives in the development of protein and enzyme mimics, see: Santos et al. (2001). For related structures, see: Zhang et al. (2006); Han et al. (2008); Guo et al. (2010). For reference bond-length data, see: Allen et al. (1987).<smiles>COc1ccc(/C=N/c2c(C)n(C)n(-c3ccccc3)c2=O)cc1OS(=O)(=O)c1ccc(Cl)cc1</smiles>

\section{Experimental}

Crystal data

$\mathrm{C}_{25} \mathrm{H}_{22} \mathrm{ClN}_{3} \mathrm{O}_{5} \mathrm{~S} \quad M_{r}=511.98$
Monoclinic, $P 2_{1} / c$

$a=11.063$ (2) А

$b=10.153(2) \AA$

$c=22.159$ (4) $\AA$

$\beta=98.73(3)^{\circ}$

$V=2460.1(8) \AA^{3}$

$Z=4$

Mo $K \alpha$ radiation

$\mu=0.28 \mathrm{~mm}^{-1}$

$T=294 \mathrm{~K}$

$0.30 \times 0.26 \times 0.18 \mathrm{~mm}$

Data collection

Bruker SMART APEX CCD areadetector diffractometer

Absorption correction: multi-scan (SADABS; Sheldrick, 1996)

$T_{\min }=0.907, T_{\max }=0.951$

Refinement

$R\left[F^{2}>2 \sigma\left(F^{2}\right)\right]=0.056$

$w R\left(F^{2}\right)=0.154$

$S=1.00$

4315 reflections

19683 measured reflections 4315 independent reflections 3016 reflections with $I>2 \sigma(I)$

$R_{\text {int }}=0.052$

Table 1

Hydrogen-bond geometry $\left(\AA{ }^{\circ}\right)$.

\begin{tabular}{lllll}
\hline$D-\mathrm{H} \cdots A$ & $D-\mathrm{H}$ & $\mathrm{H} \cdots A$ & $D \cdots A$ & $D-\mathrm{H} \cdots A$ \\
\hline $\mathrm{C} 11-\mathrm{H} 11 \cdots \mathrm{O}^{\mathrm{i}}$ & 0.93 & 2.43 & $3.199(3)$ & 140 \\
\hline
\end{tabular}

Symmetry code: (i) $-x+2,-y+1,-z$.

Data collection: SMART (Bruker, 1999); cell refinement: SAINT (Bruker, 1999); data reduction: SAINT; program(s) used to solve structure: SHELXS97 (Sheldrick, 2008); program(s) used to refine structure: SHELXL97 (Sheldrick, 2008); molecular graphics: SHELXTL (Sheldrick, 2008); software used to prepare material for publication: SHELXTL.

Supplementary data and figures for this paper are available from the IUCr electronic archives (Reference: CV5310).

\section{References}

Allen, F. H., Kennard, O., Watson, D. G., Brammer, L., Orpen, A. G. \& Taylor, R. (1987). J. Chem. Soc. Perkin Trans. 2, pp. S1-19.

Bruker (1999). SMART and SAINT. Bruker AXS Inc., Madison, Wisconsin, USA.

Guo, M.-J., Chen, X. \& Yao, J.-X. (2010). Acta Cryst. E66, o1360.

Han, J.-R., Tian, X., Zhen, X.-L., Li, Z.-C. \& Liu, S.-X. (2008). Acta Cryst. E64, o2244.

Santos, M. L. P., Bagatin, I. A., Pereira, E. M. \& Ferreira, A. M. D. C. (2001). J. Chem. Soc. Dalton Trans. pp. 838-844.

Sheldrick, G. M. (1996). SADABS. University of Göttingen, Germany.

Sheldrick, G. M. (2008). Acta Cryst. A64, 112-122.

Zhang, Q.-Z., Zhao, Y.-L., Chen, X. \& Yu, M. (2006). Acta Cryst. E62, o525205254 . 


\section{supporting information}

Acta Cryst. (2012). E68, o2110 [https://doi.org/10.1107/S160053681202627X]

(E)-5-[(1,5-Dimethyl-3-oxo-2-phenyl-2,3-dihydro-1 H-pyrazol-4-yl)iminomethyl]-2-methoxyphenyl 4-chlorobenzenesulfonate

\section{Tian-Xiang Lei}

\section{S1. Comment}

Schiff bases have extensively been studied because of their potentially biological activities such as protein and enzyme mimics (Santos et al., 2001). Among the large number of compounds, 4-amino-1,5-dimethyl-2-phenylpyrazol-3-one forms a variety of Schiff bases with aldehydes, and the synthesis and crystal structures of some of them, such as (E)-5-((1,5-Dimethyl-3-oxo-2-phenyl-2,3-dihydro-1H-pyrazol-4-ylimino)methyl) -2-methoxyphenyl 4-bromobenzenesulfonate (Guo et al., 2010), (E)-4-((1,5-Dimethyl-3-oxo-2-phenyl-2,3-dihydro-1H-pyrazol-4-ylimino)methyl) phenyl 4bromobenzenesulfonate (Han et al., 2008) and (E)-4-(2-(4-Chlorobenzyloxy)benzylideneamino) -2,3-dimethyl-1phenyl-1,2-dihydropyrazol-5-one (Zhang et al., 2006) have been reported. Herewith we report the synthesis and crystal structure of the title Schiff base compound.

In the title molecule (Fig. 1), bond lengths and angles are within normal ranges (Allen et al., 1987). Two $\mathrm{N}$ atoms in the pyrazole ring have a pyramidal environment with the sums of the valence angles around them of 349.3 (2) and $357.5(2)^{\circ}$, respectively. The phenyl ring is twisted at $50.97(12)^{\circ}$ from the pyrazole mean plane. The central benzene ring (C7-C12) with three attached atoms $(\mathrm{C} 14 / \mathrm{O} 3 / \mathrm{O} 4)$ is nearly planar, with an r.m.s. deviation for fitted atoms of $0.0392 \AA$. The mean plane of this fragment formss dihedral angles of $32.89(8)^{\circ}, 38.18(10)^{\circ}$ and $82.42(7)^{\circ}$, respectively, with the the pyrazolone ring $(\mathrm{C} 15-\mathrm{C} 17 / \mathrm{N} 1-\mathrm{N} 3 / \mathrm{O} 5)$, the chlorobenzene ring $(\mathrm{C} 1-\mathrm{C} 6)$ and the terminal phenyl ring $(\mathrm{C} 20-\mathrm{C} 25)$. Similar values of $32.02(14)^{\circ}, 37.49(18)^{\circ}$ and $80.52(13)^{\circ}$, respectively, were observed in isostructural $(E)-5-((1,5-\mathrm{Di}$ methyl-3-oxo-2-phenyl-2,3-dihydro-1 $H$-pyrazol -4-ylimino)methyl)-2-methoxyphenyl 4-bromobenzenesulfonate (Guo et al., 2010).

In the crystal, non-classical intermolecular $\mathrm{C} 11-\mathrm{H} 11 \cdots \mathrm{O} 5=\mathrm{C} 16$ hydrogen bonds (Table 1 ) form inversion-related dimers (Fig. 2).

\section{S2. Experimental}

An anhydrous ethanol solution $(100 \mathrm{ml})$ of 5-formyl-2-methoxyphenyl 4-chlorobenzenesulfonate $(3.27 \mathrm{~g}, 10 \mathrm{mmol})$ was added to an anhydrous ethanol solution $(100 \mathrm{ml})$ of 4-amino-1,5-dimethyl-2-phenylpyrazol-3-one $(2.03 \mathrm{~g}, 10 \mathrm{mmol})$ and the mixture refluxed for $3 \mathrm{~h}$ under $\mathrm{N}_{2}$, giving a yellow precipitate. The product was isolated, recrystallized from acetonitrile, and then dried in a vacuum to give pure compound (I) in 76\% yield. Yellow single crystals of the title compound suitable for X-ray analysis were obtained by slow evaporation of an acetonitrile solution.

\section{S3. Refinement}

The $\mathrm{H}$ atoms were included in calculated positions and refined using a riding model approximation. Constrained $\mathrm{C}-\mathrm{H}$ bond lengths and isotropic $U$ parameters: $0.93 \AA$ and $U_{\text {iso }}(\mathrm{H})=1.2 U_{\text {eq }}(\mathrm{C})$ for $C s p^{2}-\mathrm{H} ; 0.96 \AA$ and $U_{\text {iso }}(\mathrm{H})=1.5 U_{\text {eq }}(\mathrm{C})$ for methyl C-H. 


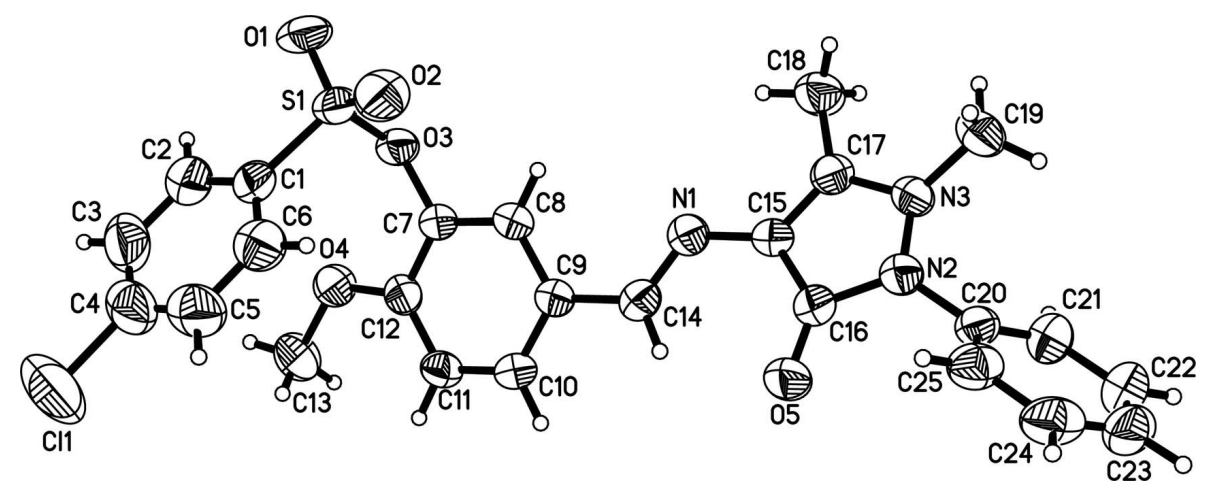

Figure 1

The structure of the title molecule, with displacement ellipsoids for non- $\mathrm{H}$ atoms drawn at the $50 \%$ probability level.

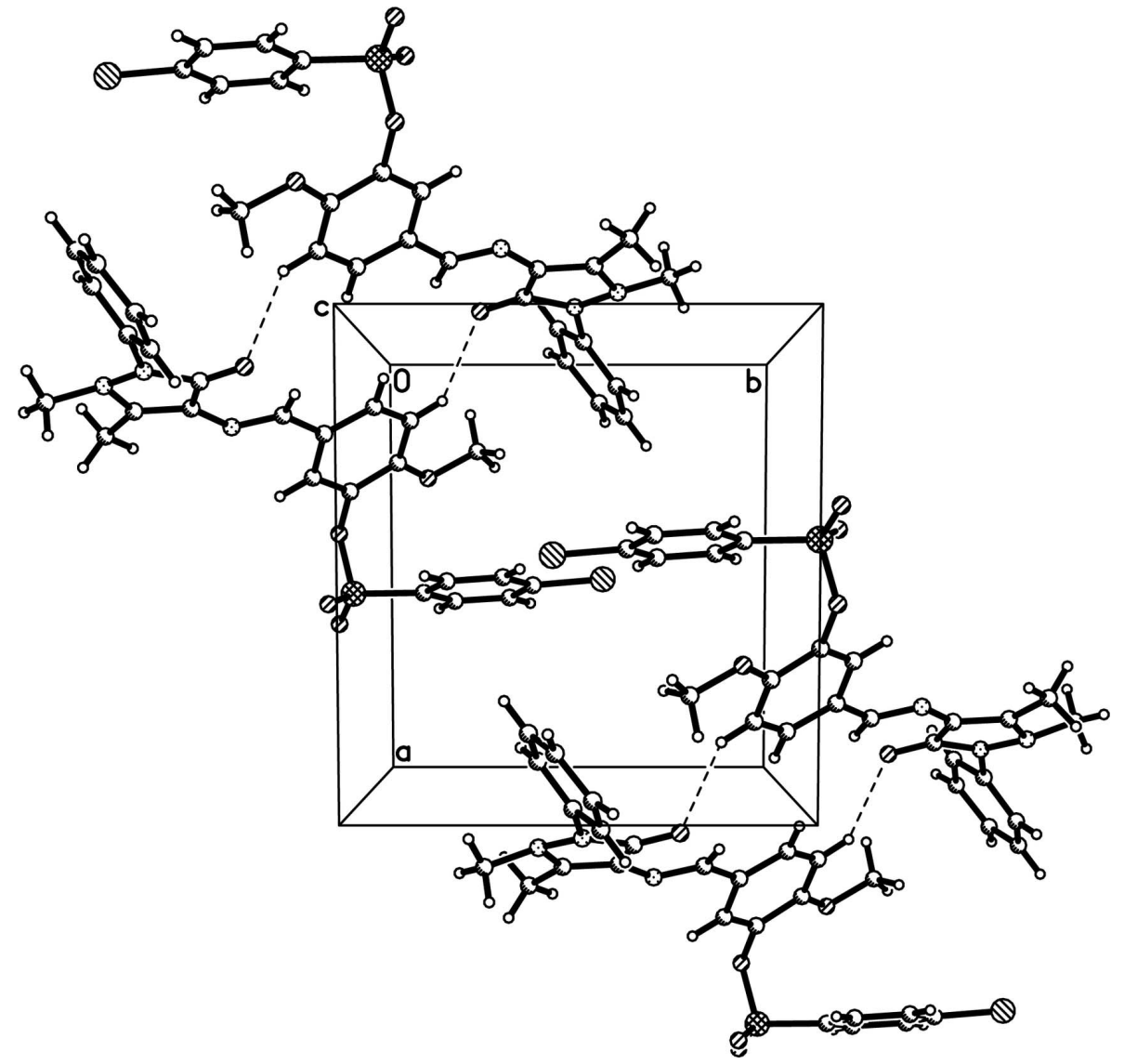

Figure 2

A portion of the crystal packing showing weak $\mathrm{C}-\mathrm{H} \cdots \mathrm{O}$ interactions as dashed lines.

(E)-5-[(1,5-Dimethyl-3-oxo-2-phenyl-2,3-dihydro-1 $H$-pyrazol-4- yl)iminomethyl]-2-methoxyphenyl 4-

chlorobenzenesulfonate

Crystal data

$\mathrm{C}_{25} \mathrm{H}_{22} \mathrm{ClN}_{3} \mathrm{O}_{5} \mathrm{~S}$

Monoclinic, $P 2_{1} / c$

$M_{r}=511.98$

Hall symbol: -P 2ybc 


$$
\begin{aligned}
& a=11.063(2) \AA \\
& b=10.153(2) \AA \\
& c=22.159(4) \AA \\
& \beta=98.73(3)^{\circ} \\
& V=2460.1(8) \AA^{3} \\
& Z=4 \\
& F(000)=1064 \\
& D_{\mathrm{x}}=1.382 \mathrm{Mg} \mathrm{m}^{-3}
\end{aligned}
$$

\section{Data collection}

Bruker SMART APEX CCD area-detector diffractometer

Radiation source: fine-focus sealed tube

Graphite monochromator

$\varphi$ and $\omega$ scans

Absorption correction: multi-scan (SADABS; Sheldrick, 1996)

$T_{\min }=0.907, T_{\max }=0.951$

\section{Refinement}

Refinement on $F^{2}$

Least-squares matrix: full

$R\left[F^{2}>2 \sigma\left(F^{2}\right)\right]=0.056$

$w R\left(F^{2}\right)=0.154$

$S=1.00$

4315 reflections

319 parameters

0 restraints

Primary atom site location: structure-invariant direct methods
Mo $K \alpha$ radiation, $\lambda=0.71073 \AA$

Cell parameters from 5316 reflections

$\theta=2.7-27.9^{\circ}$

$\mu=0.28 \mathrm{~mm}^{-1}$

$T=294 \mathrm{~K}$

Block, yellow

$0.30 \times 0.26 \times 0.18 \mathrm{~mm}$

19683 measured reflections

4315 independent reflections

3016 reflections with $I>2 \sigma(I)$

$R_{\text {int }}=0.052$

$\theta_{\text {max }}=25.0^{\circ}, \theta_{\min }=2.4^{\circ}$

$h=-11 \rightarrow 13$

$k=-12 \rightarrow 11$

$l=-26 \rightarrow 26$

Secondary atom site location: difference Fourier map

Hydrogen site location: inferred from neighbouring sites

$\mathrm{H}$-atom parameters constrained

$w=1 /\left[\sigma^{2}\left(F_{\mathrm{o}}{ }^{2}\right)+(0.0919 P)^{2}\right]$

where $P=\left(F_{\mathrm{o}}^{2}+2 F_{\mathrm{c}}^{2}\right) / 3$

$(\Delta / \sigma)_{\max }<0.001$

$\Delta \rho_{\max }=0.34$ e $\AA^{-3}$

$\Delta \rho_{\min }=-0.45$ e $\AA^{-3}$

Special details

Geometry. All e.s.d.'s (except the e.s.d. in the dihedral angle between two 1.s. planes) are estimated using the full covariance matrix. The cell e.s.d.'s are taken into account individually in the estimation of e.s.d.'s in distances, angles and torsion angles; correlations between e.s.d.'s in cell parameters are only used when they are defined by crystal symmetry. An approximate (isotropic) treatment of cell e.s.d.'s is used for estimating e.s.d.'s involving 1.s. planes.

Refinement. Refinement of $F^{2}$ against ALL reflections. The weighted $R$-factor $w R$ and goodness of fit $S$ are based on $F^{2}$, conventional $R$-factors $R$ are based on $F$, with $F$ set to zero for negative $F^{2}$. The threshold expression of $F^{2}>2 \sigma\left(F^{2}\right)$ is used only for calculating $R$-factors(gt) $e t c$. and is not relevant to the choice of reflections for refinement. $R$-factors based on $F^{2}$ are statistically about twice as large as those based on $F$, and $R$ - factors based on ALL data will be even larger.

Fractional atomic coordinates and isotropic or equivalent isotropic displacement parameters $\left(\AA^{2}\right)$

\begin{tabular}{lllll}
\hline & $x$ & $y$ & $z$ & $U_{\text {iso }} * / U_{\text {eq }}$ \\
\hline C11 & $0.47353(11)$ & $1.05947(9)$ & $0.12284(7)$ & $0.1381(5)$ \\
S1 & $0.44039(6)$ & $0.44761(7)$ & $0.13133(3)$ & $0.0563(3)$ \\
N1 & $0.80799(17)$ & $0.1811(2)$ & $0.00538(9)$ & $0.0454(5)$ \\
N2 & $0.91575(19)$ & $0.0090(2)$ & $-0.11867(10)$ & $0.0515(6)$ \\
N3 & $0.88868(18)$ & $-0.09430(19)$ & $-0.08078(9)$ & $0.0477(5)$ \\
O1 & $0.36954(18)$ & $0.4061(2)$ & $0.17629(10)$ & $0.0786(7)$ \\
O2 & $0.41778(17)$ & $0.3950(2)$ & $0.07100(9)$ & $0.0729(6)$ \\
O3 & $0.57713(14)$ & $0.40946(15)$ & $0.16147(7)$ & $0.0497(5)$ \\
O4 & $0.70559(15)$ & $0.62701(17)$ & $0.19204(8)$ & $0.0548(5)$
\end{tabular}




\begin{tabular}{|c|c|c|c|c|}
\hline O5 & $0.92591(17)$ & $0.23713(16)$ & $-0.11241(9)$ & $0.0617(5)$ \\
\hline $\mathrm{C} 1$ & $0.4426(2)$ & $0.6207(3)$ & $0.12761(12)$ & $0.0516(7)$ \\
\hline $\mathrm{C} 2$ & $0.4230(3)$ & $0.6931(3)$ & $0.17786(15)$ & $0.0699(8)$ \\
\hline $\mathrm{H} 2$ & 0.4046 & 0.6510 & 0.2126 & $0.084 *$ \\
\hline $\mathrm{C} 3$ & $0.4310(3)$ & $0.8292(3)$ & $0.17608(19)$ & $0.0835(10)$ \\
\hline $\mathrm{H} 3$ & 0.4171 & 0.8797 & 0.2094 & $0.100 *$ \\
\hline $\mathrm{C} 4$ & $0.4600(3)$ & $0.8884(3)$ & $0.1241(2)$ & $0.0829(10)$ \\
\hline $\mathrm{C} 5$ & $0.4784(3)$ & $0.8173(4)$ & $0.07370(18)$ & $0.0809(10)$ \\
\hline H5 & 0.4966 & 0.8596 & 0.0389 & $0.097^{*}$ \\
\hline C6 & $0.4693(2)$ & $0.6812(3)$ & $0.07553(14)$ & $0.0651(8)$ \\
\hline H6 & 0.4812 & 0.6311 & 0.0418 & $0.078^{*}$ \\
\hline $\mathrm{C} 7$ & $0.67271(19)$ & $0.4424(2)$ & $0.12844(10)$ & $0.0406(6)$ \\
\hline $\mathrm{C} 8$ & $0.7020(2)$ & $0.3589(2)$ & $0.08434(11)$ & $0.0440(6)$ \\
\hline $\mathrm{H} 8$ & 0.6593 & 0.2804 & 0.0761 & $0.053 *$ \\
\hline C9 & $0.7967(2)$ & $0.3923(2)$ & $0.05159(11)$ & $0.0426(6)$ \\
\hline $\mathrm{C} 10$ & $0.8574(2)$ & $0.5106(2)$ & $0.06549(11)$ & $0.0480(6)$ \\
\hline $\mathrm{H} 10$ & 0.9176 & 0.5362 & 0.0428 & $0.058^{*}$ \\
\hline $\mathrm{C} 11$ & $0.8311(2)$ & $0.5919(2)$ & $0.11203(11)$ & $0.0462(6)$ \\
\hline H11 & 0.8754 & 0.6690 & 0.1213 & $0.055^{*}$ \\
\hline $\mathrm{C} 12$ & $0.7384(2)$ & $0.5579(2)$ & $0.14468(11)$ & $0.0425(6)$ \\
\hline $\mathrm{C} 13$ & $0.7683(3)$ & $0.7483(3)$ & $0.20859(15)$ & $0.0705(9)$ \\
\hline H13A & 0.8534 & 0.7307 & 0.2222 & $0.106^{*}$ \\
\hline H13B & 0.7334 & 0.7898 & 0.2409 & $0.106^{*}$ \\
\hline $\mathrm{H} 13 \mathrm{C}$ & 0.7602 & 0.8058 & 0.1738 & $0.106^{*}$ \\
\hline $\mathrm{C} 14$ & $0.8334(2)$ & $0.3028(3)$ & $0.00548(11)$ & $0.0479(6)$ \\
\hline H14 & 0.8762 & 0.3361 & -0.0242 & $0.057 *$ \\
\hline $\mathrm{C} 15$ & $0.8452(2)$ & $0.0964(2)$ & $-0.03798(11)$ & $0.0426(6)$ \\
\hline $\mathrm{C} 16$ & $0.8973(2)$ & $0.1298(2)$ & $-0.09185(11)$ & $0.0464(6)$ \\
\hline $\mathrm{C} 17$ & $0.8386(2)$ & $-0.0375(2)$ & $-0.03448(11)$ & $0.0454(6)$ \\
\hline $\mathrm{C} 18$ & $0.7882(3)$ & $-0.1191(3)$ & $0.01137(13)$ & $0.0641(8)$ \\
\hline H18A & 0.7636 & -0.0631 & 0.0422 & $0.096^{*}$ \\
\hline H18B & 0.8497 & -0.1794 & 0.0299 & $0.096^{*}$ \\
\hline $\mathrm{H} 18 \mathrm{C}$ & 0.7188 & -0.1676 & -0.0083 & $0.096^{*}$ \\
\hline C19 & 0.8505 & $-0.2198(3)$ & $-0.10901(15)$ & $0.0740(9)$ \\
\hline H19A & 0.8321 & -0.2801 & -0.0783 & $0.111^{*}$ \\
\hline H19B & 0.9152 & -0.2551 & -0.1285 & $0.111^{*}$ \\
\hline $\mathrm{H} 19 \mathrm{C}$ & 0.7790 & -0.2070 & -0.1389 & $0.111^{*}$ \\
\hline $\mathrm{C} 20$ & $0.9860(2)$ & $-0.0126(2)$ & $-0.16676(11)$ & $0.0464(6)$ \\
\hline $\mathrm{C} 21$ & $1.0877(2)$ & $-0.0934(3)$ & $-0.15858(13)$ & $0.0590(7)$ \\
\hline $\mathrm{H} 21$ & 1.1128 & -0.1339 & -0.1212 & $0.071 *$ \\
\hline $\mathrm{C} 22$ & $1.1517(3)$ & $-0.1131(3)$ & $-0.20709(15)$ & $0.0713(9)$ \\
\hline $\mathrm{H} 22$ & 1.2197 & -0.1681 & -0.2022 & $0.086^{*}$ \\
\hline $\mathrm{C} 23$ & $1.1156(3)$ & $-0.0521(3)$ & $-0.26230(15)$ & $0.0746(10)$ \\
\hline $\mathrm{H} 23$ & 1.1578 & -0.0676 & -0.2949 & $0.090^{*}$ \\
\hline $\mathrm{C} 24$ & $1.0169(3)$ & $0.0320(3)$ & $-0.26911(13)$ & $0.0698(9)$ \\
\hline $\mathrm{H} 24$ & 0.9948 & 0.0764 & -0.3058 & $0.084 *$ \\
\hline $\mathrm{C} 25$ & $0.9499(2)$ & $0.0510(3)$ & $-0.22172(12)$ & $0.0581(7)$ \\
\hline $\mathrm{H} 25$ & 0.8817 & 0.1057 & -0.2268 & $0.070^{*}$ \\
\hline
\end{tabular}


Atomic displacement parameters $\left(\AA^{2}\right)$

\begin{tabular}{|c|c|c|c|c|c|c|}
\hline & $U^{11}$ & $U^{22}$ & $U^{33}$ & $U^{12}$ & $U^{13}$ & $U^{23}$ \\
\hline $\mathrm{Cl1}$ & $0.1248(9)$ & $0.0543(6)$ & $0.2232(15)$ & $0.0018(5)$ & $-0.0119(9)$ & $0.0116(7)$ \\
\hline $\mathrm{S} 1$ & $0.0484(4)$ & $0.0558(5)$ & $0.0687(5)$ & $-0.0162(3)$ & 0.0215 & $-0.0118(3)$ \\
\hline N1 & $0.0461(11)$ & $0.0448(13)$ & $0.0460(12)$ & $-0.0022(10)$ & $0.0092(9)$ & $-0.0025(9)$ \\
\hline N2 & $0.0614(13)$ & $0.0431(12)$ & $0.0546(14)$ & $-0.0067(10)$ & $0.0239(11)$ & $-0.0020(10)$ \\
\hline N3 & $0.0568(12)$ & $0.0386(12)$ & $0.0500(12)$ & $-0.0078(10)$ & $0.0156(10)$ & $-0.0030(9)$ \\
\hline $\mathrm{O} 1$ & $0.0710(13)$ & $0.0767(14)$ & $0.0990(16)$ & $-0.0271(11)$ & $0.0480(12)$ & $-0.0073(12)$ \\
\hline $\mathrm{O} 2$ & $0.0650(12)$ & $0.0821(15)$ & $0.0712(14)$ & $-0.0193(10)$ & $0.0095(10)$ & $-0.0306(11)$ \\
\hline $\mathrm{O} 3$ & $0.0537(10)$ & $0.0459(10)$ & $0.0535(11)$ & $-0.0081(8)$ & $0.0208(8)$ & $0.0004(8)$ \\
\hline $\mathrm{O} 4$ & $0.0549(11)$ & $0.0530(11)$ & $0.0576(11)$ & $-0.0096(8)$ & $0.0124(9)$ & $-0.0172(8)$ \\
\hline $\mathrm{O} 5$ & $0.0811(13)$ & $0.0442(11)$ & $0.0669(13)$ & $-0.0084(9)$ & $0.0345(11)$ & $0.0020(9)$ \\
\hline $\mathrm{C} 1$ & $0.0391(14)$ & $0.0532(16)$ & $0.0631(17)$ & $-0.0016(11)$ & $0.0102(12)$ & -0.0045 \\
\hline $\mathrm{C} 2$ & $0.0656(18)$ & $0.065(2)$ & $0.083(2)$ & $-0.0015(16)$ & $0.0242(16)$ & $-0.0099(17)$ \\
\hline C3 & $0.073(2)$ & $0.066(2)$ & $0.111(3)$ & 0.0068 (17) & $0.010(2)$ & $-0.026(2)$ \\
\hline $\mathrm{C} 4$ & 0.0586 (19) & $0.058(2)$ & $0.126(3)$ & $0.0053(16)$ & $-0.006(2)$ & $0.009(2)$ \\
\hline $\mathrm{C} 5$ & $0.067(2)$ & $0.073(2)$ & $0.099(3)$ & $0.0016(17)$ & $-0.0010(19)$ & $0.026(2)$ \\
\hline C6 & $0.0547(17)$ & $0.069(2)$ & $0.069(2)$ & $0.0006(14)$ & $-0.0002(14)$ & $0.0035(15)$ \\
\hline $\mathrm{C} 7$ & $0.0411(13)$ & $0.0379(13)$ & $0.0448(14)$ & $-0.0021(10)$ & 0.0125 & $0.0016(10)$ \\
\hline $\mathrm{C} 8$ & $0.0463(13)$ & $0.0356(13)$ & $0.0512(15)$ & $-0.0052(11)$ & $0.0111(11)$ & $0.0004(11)$ \\
\hline C9 & $0.0453(13)$ & $0.0372(13)$ & 0.0469 (14) & $-0.0023(11)$ & $0.0122(11)$ & $0.0010(10)$ \\
\hline $\mathrm{C} 10$ & $0.0462(14)$ & $0.0453(15)$ & $0.0558(16)$ & $-0.0061(11)$ & $0.0184(12)$ & $0.0029(12)$ \\
\hline C11 & $0.0426(13)$ & 0.0395 (14) & $0.0560(16)$ & $-0.0081(11)$ & $0.0061(11)$ & $-0.0025(11)$ \\
\hline $\mathrm{C} 12$ & $0.0423(14)$ & $0.0383(13)$ & $0.0458(14)$ & $0.0016(11)$ & $0.0034(11)$ & $-0.0020(11)$ \\
\hline C13 & $0.0780(19)$ & $0.0533(18)$ & $0.081(2)$ & $-0.0145(15)$ & $0.0125(16)$ & $-0.0280(15)$ \\
\hline C14 & $0.0472(14)$ & $0.0502(16)$ & $0.0491(15)$ & $-0.0022(12)$ & $0.0163(12)$ & $-0.0024(12)$ \\
\hline C15 & $0.0400(13)$ & $0.0410(14)$ & $0.0476(14)$ & $-0.0054(11)$ & $0.0095(11)$ & $-0.0009(11)$ \\
\hline C16 & $0.0429(13)$ & $0.0451(15)$ & $0.0530(15)$ & $-0.0036(11)$ & $0.0131(11)$ & $-0.0041(12)$ \\
\hline C17 & $0.0429(14)$ & $0.0480(15)$ & $0.0462(15)$ & $-0.0034(11)$ & $0.0098(11)$ & $0.0003(11)$ \\
\hline C18 & $0.080(2)$ & $0.0533(17)$ & 0.0636 (19) & $-0.0114(14)$ & $0.0245(16)$ & $0.0040(14)$ \\
\hline C19 & $0.094(2)$ & $0.0513(18)$ & $0.083(2)$ & $-0.0229(16)$ & 0.0352 (19) & $-0.0200(16)$ \\
\hline $\mathrm{C} 20$ & $0.0508(15)$ & $0.0472(15)$ & $0.0439(14)$ & $-0.0112(12)$ & $0.0153(11)$ & $-0.0061(11)$ \\
\hline C21 & $0.0636(17)$ & $0.0621(18)$ & $0.0544(17)$ & $0.0006(14)$ & $0.0187(14)$ & $-0.0044(13)$ \\
\hline $\mathrm{C} 22$ & $0.072(2)$ & $0.071(2)$ & $0.078(2)$ & $0.0013(16)$ & $0.0342(17)$ & $-0.0096(17)$ \\
\hline $\mathrm{C} 23$ & $0.090(2)$ & $0.080(2)$ & $0.063(2)$ & $-0.0271(19)$ & 0.0385 & $-0.0237(17)$ \\
\hline $\mathrm{C} 24$ & $0.089(2)$ & $0.078(2)$ & $0.0429(16)$ & $-0.0328(19)$ & $0.0130(15)$ & $-0.0034(14)$ \\
\hline $\mathrm{C} 25$ & $0.0553(16)$ & 0.0638 (19) & $0.0547(17)$ & $-0.0154(13)$ & $0.0066(13)$ & $-0.0030(14)$ \\
\hline
\end{tabular}

Geometric parameters ( $\AA, \stackrel{\circ}{)})$

\begin{tabular}{llll}
\hline $\mathrm{C} 11-\mathrm{C} 4$ & $1.743(3)$ & $\mathrm{C} 9-\mathrm{C} 10$ & $1.388(3)$ \\
$\mathrm{S} 1-\mathrm{O} 1$ & $1.4218(18)$ & $\mathrm{C} 9-\mathrm{C} 14$ & $1.470(3)$ \\
$\mathrm{S} 1-\mathrm{O} 2$ & $1.4263(19)$ & $\mathrm{C} 10-\mathrm{C} 11$ & $1.386(3)$ \\
$\mathrm{S} 1-\mathrm{O} 3$ & $1.6055(18)$ & $\mathrm{C} 10-\mathrm{H} 10$ & 0.9300 \\
$\mathrm{~S} 1-\mathrm{C} 1$ & $1.760(3)$ & $\mathrm{C} 11-\mathrm{C} 12$ & $1.385(3)$ \\
$\mathrm{N} 1-\mathrm{C} 14$ & $1.267(3)$ & $\mathrm{C} 11-\mathrm{H} 11$ & 0.9300 \\
$\mathrm{~N} 1-\mathrm{C} 15$ & $1.397(3)$ & $\mathrm{C} 13-\mathrm{H} 13 \mathrm{~A}$ & 0.9600
\end{tabular}




\begin{tabular}{|c|c|c|c|}
\hline $\mathrm{N} 2-\mathrm{C} 16$ & $1.391(3)$ & $\mathrm{C} 13-\mathrm{H} 13 \mathrm{~B}$ & 0.9600 \\
\hline $\mathrm{N} 2-\mathrm{N} 3$ & $1.404(3)$ & $\mathrm{C} 13-\mathrm{H} 13 \mathrm{C}$ & 0.9600 \\
\hline $\mathrm{N} 2-\mathrm{C} 20$ & $1.428(3)$ & $\mathrm{C} 14-\mathrm{H} 14$ & 0.9300 \\
\hline $\mathrm{N} 3-\mathrm{C} 17$ & $1.365(3)$ & $\mathrm{C} 15-\mathrm{C} 17$ & $1.365(3)$ \\
\hline N3- 19 & $1.453(3)$ & $\mathrm{C} 15-\mathrm{C} 16$ & $1.443(3)$ \\
\hline $\mathrm{O} 3-\mathrm{C} 7$ & $1.414(2)$ & $\mathrm{C} 17-\mathrm{C} 18$ & $1.484(3)$ \\
\hline $\mathrm{O} 4-\mathrm{C} 12$ & $1.357(3)$ & $\mathrm{C} 18-\mathrm{H} 18 \mathrm{~A}$ & 0.9600 \\
\hline $\mathrm{O} 4-\mathrm{C} 13$ & $1.434(3)$ & C18-H18B & 0.9600 \\
\hline $\mathrm{O} 5-\mathrm{C} 16$ & $1.241(3)$ & $\mathrm{C} 18-\mathrm{H} 18 \mathrm{C}$ & 0.9600 \\
\hline $\mathrm{C} 1-\mathrm{C} 2$ & $1.379(4)$ & C19-H19A & 0.9600 \\
\hline $\mathrm{C} 1-\mathrm{C} 6$ & $1.379(4)$ & C19-H19B & 0.9600 \\
\hline $\mathrm{C} 2-\mathrm{C} 3$ & $1.386(4)$ & $\mathrm{C} 19-\mathrm{H} 19 \mathrm{C}$ & 0.9600 \\
\hline $\mathrm{C} 2-\mathrm{H} 2$ & 0.9300 & $\mathrm{C} 20-\mathrm{C} 21$ & $1.381(4)$ \\
\hline $\mathrm{C} 3-\mathrm{C} 4$ & $1.379(5)$ & $\mathrm{C} 20-\mathrm{C} 25$ & $1.383(3)$ \\
\hline $\mathrm{C} 3-\mathrm{H} 3$ & 0.9300 & $\mathrm{C} 21-\mathrm{C} 22$ & $1.389(3)$ \\
\hline $\mathrm{C} 4-\mathrm{C} 5$ & $1.371(5)$ & $\mathrm{C} 21-\mathrm{H} 21$ & 0.9300 \\
\hline $\mathrm{C} 5-\mathrm{C} 6$ & $1.386(5)$ & $\mathrm{C} 22-\mathrm{C} 23$ & $1.375(4)$ \\
\hline $\mathrm{C} 5-\mathrm{H} 5$ & 0.9300 & $\mathrm{C} 22-\mathrm{H} 22$ & 0.9300 \\
\hline $\mathrm{C} 6-\mathrm{H} 6$ & 0.9300 & $\mathrm{C} 23-\mathrm{C} 24$ & $1.377(4)$ \\
\hline $\mathrm{C} 7-\mathrm{C} 8$ & $1.370(3)$ & $\mathrm{C} 23-\mathrm{H} 23$ & 0.9300 \\
\hline $\mathrm{C} 7-\mathrm{C} 12$ & $1.397(3)$ & $\mathrm{C} 24-\mathrm{C} 25$ & $1.388(4)$ \\
\hline $\mathrm{C} 8-\mathrm{C} 9$ & $1.404(3)$ & $\mathrm{C} 24-\mathrm{H} 24$ & 0.9300 \\
\hline $\mathrm{C} 8-\mathrm{H} 8$ & 0.9300 & $\mathrm{C} 25-\mathrm{H} 25$ & 0.9300 \\
\hline $\mathrm{O} 1-\mathrm{S} 1-\mathrm{O} 2$ & $120.58(13)$ & $\mathrm{C} 11-\mathrm{C} 12-\mathrm{C} 7$ & $118.2(2)$ \\
\hline $\mathrm{O} 1-\mathrm{S} 1-\mathrm{O} 3$ & $102.82(12)$ & $\mathrm{O} 4-\mathrm{C} 13-\mathrm{H} 13 \mathrm{~A}$ & 109.5 \\
\hline $\mathrm{O} 2-\mathrm{S} 1-\mathrm{O} 3$ & $108.64(11)$ & $\mathrm{O} 4-\mathrm{C} 13-\mathrm{H} 13 \mathrm{~B}$ & 109.5 \\
\hline $\mathrm{O} 1-\mathrm{S} 1-\mathrm{C} 1$ & $110.01(12)$ & $\mathrm{H} 13 \mathrm{~A}-\mathrm{C} 13-\mathrm{H} 13 \mathrm{~B}$ & 109.5 \\
\hline $\mathrm{O} 2-\mathrm{S} 1-\mathrm{C} 1$ & $109.40(14)$ & $\mathrm{O} 4-\mathrm{C} 13-\mathrm{H} 13 \mathrm{C}$ & 109.5 \\
\hline $\mathrm{O} 3-\mathrm{S} 1-\mathrm{C} 1$ & $103.96(10)$ & $\mathrm{H} 13 \mathrm{~A}-\mathrm{C} 13-\mathrm{H} 13 \mathrm{C}$ & 109.5 \\
\hline $\mathrm{C} 14-\mathrm{N} 1-\mathrm{C} 15$ & $120.9(2)$ & $\mathrm{H} 13 \mathrm{~B}-\mathrm{C} 13-\mathrm{H} 13 \mathrm{C}$ & 109.5 \\
\hline $\mathrm{C} 16-\mathrm{N} 2-\mathrm{N} 3$ & $110.16(19)$ & $\mathrm{N} 1-\mathrm{C} 14-\mathrm{C} 9$ & $121.3(2)$ \\
\hline $\mathrm{C} 16-\mathrm{N} 2-\mathrm{C} 20$ & $125.9(2)$ & $\mathrm{N} 1-\mathrm{C} 14-\mathrm{H} 14$ & 119.4 \\
\hline $\mathrm{N} 3-\mathrm{N} 2-\mathrm{C} 20$ & $121.39(19)$ & $\mathrm{C} 9-\mathrm{C} 14-\mathrm{H} 14$ & 119.4 \\
\hline $\mathrm{C} 17-\mathrm{N} 3-\mathrm{N} 2$ & $106.35(19)$ & $\mathrm{C} 17-\mathrm{C} 15-\mathrm{N} 1$ & $123.4(2)$ \\
\hline $\mathrm{C} 17-\mathrm{N} 3-\mathrm{C} 19$ & $124.7(2)$ & $\mathrm{C} 17-\mathrm{C} 15-\mathrm{C} 16$ & $108.2(2)$ \\
\hline $\mathrm{N} 2-\mathrm{N} 3-\mathrm{C} 19$ & $118.2(2)$ & $\mathrm{N} 1-\mathrm{C} 15-\mathrm{C} 16$ & $128.3(2)$ \\
\hline $\mathrm{C} 7-\mathrm{O} 3-\mathrm{S} 1$ & $117.31(14)$ & $\mathrm{O} 5-\mathrm{C} 16-\mathrm{N} 2$ & $123.7(2)$ \\
\hline $\mathrm{C} 12-\mathrm{O} 4-\mathrm{C} 13$ & $117.80(19)$ & $\mathrm{O} 5-\mathrm{C} 16-\mathrm{C} 15$ & $131.8(2)$ \\
\hline $\mathrm{C} 2-\mathrm{C} 1-\mathrm{C} 6$ & $121.2(3)$ & $\mathrm{N} 2-\mathrm{C} 16-\mathrm{C} 15$ & $104.4(2)$ \\
\hline $\mathrm{C} 2-\mathrm{C} 1-\mathrm{S} 1$ & $119.3(2)$ & $\mathrm{C} 15-\mathrm{C} 17-\mathrm{N} 3$ & $110.3(2)$ \\
\hline $\mathrm{C} 6-\mathrm{C} 1-\mathrm{S} 1$ & $119.4(2)$ & $\mathrm{C} 15-\mathrm{C} 17-\mathrm{C} 18$ & $128.6(2)$ \\
\hline $\mathrm{C} 1-\mathrm{C} 2-\mathrm{C} 3$ & $119.4(3)$ & $\mathrm{N} 3-\mathrm{C} 17-\mathrm{C} 18$ & $121.1(2)$ \\
\hline $\mathrm{C} 1-\mathrm{C} 2-\mathrm{H} 2$ & 120.3 & $\mathrm{C} 17-\mathrm{C} 18-\mathrm{H} 18 \mathrm{~A}$ & 109.5 \\
\hline $\mathrm{C} 3-\mathrm{C} 2-\mathrm{H} 2$ & 120.3 & $\mathrm{C} 17-\mathrm{C} 18-\mathrm{H} 18 \mathrm{~B}$ & 109.5 \\
\hline $\mathrm{C} 4-\mathrm{C} 3-\mathrm{C} 2$ & $118.9(3)$ & $\mathrm{H} 18 \mathrm{~A}-\mathrm{C} 18-\mathrm{H} 18 \mathrm{~B}$ & 109.5 \\
\hline $\mathrm{C} 4-\mathrm{C} 3-\mathrm{H} 3$ & 120.6 & $\mathrm{C} 17-\mathrm{C} 18-\mathrm{H} 18 \mathrm{C}$ & 109.5 \\
\hline $\mathrm{C} 2-\mathrm{C} 3-\mathrm{H} 3$ & 120.6 & $\mathrm{H} 18 \mathrm{~A}-\mathrm{C} 18-\mathrm{H} 18 \mathrm{C}$ & 109.5 \\
\hline
\end{tabular}




\begin{tabular}{|c|c|c|c|}
\hline $\mathrm{C} 5-\mathrm{C} 4-\mathrm{C} 3$ & $122.2(3)$ & $\mathrm{H} 18 \mathrm{~B}-\mathrm{C} 18-\mathrm{H} 18 \mathrm{C}$ & 109.5 \\
\hline $\mathrm{C} 5-\mathrm{C} 4-\mathrm{Cl1}$ & $119.1(3)$ & N3-C19-H19A & 109.5 \\
\hline $\mathrm{C} 3-\mathrm{C} 4-\mathrm{Cl1}$ & $118.7(3)$ & N3-C19-H19B & 109.5 \\
\hline $\mathrm{C} 4-\mathrm{C} 5-\mathrm{C} 6$ & $118.7(3)$ & $\mathrm{H} 19 \mathrm{~A}-\mathrm{C} 19-\mathrm{H} 19 \mathrm{~B}$ & 109.5 \\
\hline $\mathrm{C} 4-\mathrm{C} 5-\mathrm{H} 5$ & 120.6 & $\mathrm{~N} 3-\mathrm{C} 19-\mathrm{H} 19 \mathrm{C}$ & 109.5 \\
\hline $\mathrm{C} 6-\mathrm{C} 5-\mathrm{H} 5$ & 120.6 & $\mathrm{H} 19 \mathrm{~A}-\mathrm{C} 19-\mathrm{H} 19 \mathrm{C}$ & 109.5 \\
\hline $\mathrm{C} 1-\mathrm{C} 6-\mathrm{C} 5$ & $119.6(3)$ & $\mathrm{H} 19 \mathrm{~B}-\mathrm{C} 19-\mathrm{H} 19 \mathrm{C}$ & 109.5 \\
\hline $\mathrm{C} 1-\mathrm{C} 6-\mathrm{H} 6$ & 120.2 & $\mathrm{C} 21-\mathrm{C} 20-\mathrm{C} 25$ & $120.9(2)$ \\
\hline $\mathrm{C} 5-\mathrm{C} 6-\mathrm{H} 6$ & 120.2 & $\mathrm{C} 21-\mathrm{C} 20-\mathrm{N} 2$ & $121.2(2)$ \\
\hline $\mathrm{C} 8-\mathrm{C} 7-\mathrm{C} 12$ & $122.3(2)$ & $\mathrm{C} 25-\mathrm{C} 20-\mathrm{N} 2$ & $117.9(2)$ \\
\hline $\mathrm{C} 8-\mathrm{C} 7-\mathrm{O} 3$ & $119.9(2)$ & $\mathrm{C} 20-\mathrm{C} 21-\mathrm{C} 22$ & $119.0(3)$ \\
\hline $\mathrm{C} 12-\mathrm{C} 7-\mathrm{O} 3$ & $117.74(19)$ & $\mathrm{C} 20-\mathrm{C} 21-\mathrm{H} 21$ & 120.5 \\
\hline $\mathrm{C} 7-\mathrm{C} 8-\mathrm{C} 9$ & $119.7(2)$ & $\mathrm{C} 22-\mathrm{C} 21-\mathrm{H} 21$ & 120.5 \\
\hline $\mathrm{C} 7-\mathrm{C} 8-\mathrm{H} 8$ & 120.2 & $\mathrm{C} 23-\mathrm{C} 22-\mathrm{C} 21$ & $120.7(3)$ \\
\hline $\mathrm{C} 9-\mathrm{C} 8-\mathrm{H} 8$ & 120.2 & $\mathrm{C} 23-\mathrm{C} 22-\mathrm{H} 22$ & 119.7 \\
\hline $\mathrm{C} 10-\mathrm{C} 9-\mathrm{C} 8$ & $117.9(2)$ & $\mathrm{C} 21-\mathrm{C} 22-\mathrm{H} 22$ & 119.7 \\
\hline $\mathrm{C} 10-\mathrm{C} 9-\mathrm{C} 14$ & $121.0(2)$ & $\mathrm{C} 22-\mathrm{C} 23-\mathrm{C} 24$ & $119.7(3)$ \\
\hline $\mathrm{C} 8-\mathrm{C} 9-\mathrm{C} 14$ & $121.1(2)$ & $\mathrm{C} 22-\mathrm{C} 23-\mathrm{H} 23$ & 120.2 \\
\hline $\mathrm{C} 11-\mathrm{C} 10-\mathrm{C} 9$ & $122.1(2)$ & $\mathrm{C} 24-\mathrm{C} 23-\mathrm{H} 23$ & 120.2 \\
\hline $\mathrm{C} 11-\mathrm{C} 10-\mathrm{H} 10$ & 118.9 & $\mathrm{C} 23-\mathrm{C} 24-\mathrm{C} 25$ & $120.6(3)$ \\
\hline $\mathrm{C} 9-\mathrm{C} 10-\mathrm{H} 10$ & 118.9 & $\mathrm{C} 23-\mathrm{C} 24-\mathrm{H} 24$ & 119.7 \\
\hline $\mathrm{C} 12-\mathrm{C} 11-\mathrm{C} 10$ & $119.7(2)$ & $\mathrm{C} 25-\mathrm{C} 24-\mathrm{H} 24$ & 119.7 \\
\hline $\mathrm{C} 12-\mathrm{C} 11-\mathrm{H} 11$ & 120.1 & $\mathrm{C} 20-\mathrm{C} 25-\mathrm{C} 24$ & $119.0(3)$ \\
\hline $\mathrm{C} 10-\mathrm{C} 11-\mathrm{H} 11$ & 120.1 & $\mathrm{C} 20-\mathrm{C} 25-\mathrm{H} 25$ & 120.5 \\
\hline $\mathrm{O} 4-\mathrm{C} 12-\mathrm{C} 11$ & $125.5(2)$ & $\mathrm{C} 24-\mathrm{C} 25-\mathrm{H} 25$ & 120.5 \\
\hline $\mathrm{O} 4-\mathrm{C} 12-\mathrm{C} 7$ & $116.35(19)$ & & \\
\hline
\end{tabular}

Hydrogen-bond geometry $\left(\AA,{ }^{\circ}\right)$

\begin{tabular}{lllll}
\hline$D-\mathrm{H} \cdots A$ & $D-\mathrm{H}$ & $\mathrm{H} \cdots A$ & $D \cdots A$ & $D-\mathrm{H} \cdots A$ \\
\hline $\mathrm{C} 11-\mathrm{H} 11 \cdots \mathrm{O}^{\mathrm{i}}$ & 0.93 & 2.43 & $3.199(3)$ & 140 \\
\hline
\end{tabular}

Symmetry code: (i) $-x+2,-y+1,-z$. 